\title{
A holistic approach to targeting disease with polymer nanoparticles
}

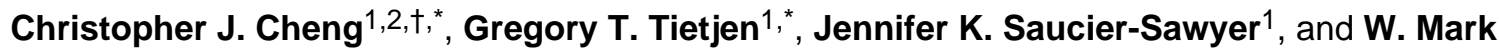 \\ Saltzman ${ }^{1}$ \\ ${ }^{1}$ Department of Biomedical Engineering, Yale University, New Haven, CT 06511, USA \\ ${ }^{2}$ Department of Molecular, Cellular and Developmental Biology, Yale University, New Haven, CT \\ 06511, USA
}

\section{PREFACE}

The primary goal of nanomedicine is to improve clinical outcomes. Toward this end, targeted nanoparticles are engineered to reduce non-productive distribution while improving diagnostic and therapeutic efficacy. Paradoxically, as this field has matured, the notion of 'targeting' has been minimized to the concept of increasing affinity of a nanoparticle for its target. This review outlines a holistic view of nanoparticle targeting, in which nanoparticle route of administration, molecular characteristics, and temporal control are potential design variables that must be considered simultaneously. This comprehensive vision for nanoparticle targeting will hasten the integration of nanomedicines into clinical practice.

\section{INTRODUCTION}

The central promise of targeted drug delivery technologies is improved efficacy by increasing drug concentration at a desired (or target) site, while simultaneously minimizing toxicity by reducing off target accumulation. The last several years have seen the development of an enormous array of systems engineered to fulfill this drug targeting promise. Such systems range from the conjugation of a hydrophilic polymer to a hydrophobic drug ${ }^{1}$, up to more complex nanocarrier systems that can dynamically respond to local environmental cues ${ }^{2}$. Ultimately, the utility of any drug delivery system-regardless of the materials used or mechanism of action-should be judged with respect to the definition of targeting; i.e. does the targeting system significantly improve efficacy and reduce toxicity by providing control over the drug biodistribution and pharmacokinetics. Many current approaches for targeted drug delivery systems assume that the best mechanism for controlling the fate of a therapeutic agent is via hijacking cellular receptor-ligand interactions. Often less consideration is given to other factors that can dramatically affect the ability to control the delivery of a drug to a specific site, properties such as: route of administration, the surface adsorption of serum proteins, drug release kinetics, and biological timing. In order to realize the full potential of targeted drug delivery, we believe

\footnotetext{
$\dagger$ Present address: Alexion Pharmaceuticals, Inc., Cheshire, CT 06410, USA

*Denotes co-first authorship
} 
that targeting should be more comprehensively defined to also include these non-canonical aspects. A holistic view of targeting encompasses all aspects of delivery from the macroscale, e.g. where and how the therapeutic is introduced into the body, to the micro-scale, e.g. the molecular interactions that govern how a delivery system interacts with cells and the extracellular milieu (Figure 1).

More than any other class of drug delivery vehicle, polymer-based nanoparticles have the capacity to fully realize on this holistic view of targeting. Polymer nanoparticles (with a sub-300 nm diameter) are structurally defined as solid nanoparticles, micelles, polyplexes, or dendrimers (Box 1). These colloidal polymer systems have gained considerable commercial and translational attention in large part due to their improved stability, biocompatibility, and potential for extended drug release kinetics compared to nonpolymeric nanosystems ${ }^{3}$. Additionally, polymeric nanoparticles provide versatility via the use of polymers of different chemical composition, hydrophilic-lipophilic balance, charge, physical structure, etc. As a result of this adaptability, nanoparticles can be formulated to deliver a range of drugs and should be adaptable to many clinical settings. Moreover, the ability to control the degradation or disassembly of polymeric nanoparticles imparts the ability to control temporal aspects of drug delivery over a wider range than permitted by other forms of nanoparticles. This diversity of potential applications makes polymeric nanoparticles attractive as therapeutic delivery vehicles. However, for each new particle formulation, this diversity must be matched with a comprehensive understanding of how both the biology of the target disease and the properties of a nanoparticle therapeutic influence delivery.

\section{Box 1}

\section{Classes of polymer nanoparticles}

Solid nanoparticles are composed of a dense polymer matrix typically stabilized by hydrophobic interactions of the constituent polymer(s). A key advantage of these systems is the ability for controlled release of various cargo ranging from hydrophobic small molecules to large proteins. BIND-014 is a solid PLA nanoparticle formulation (synthesized using an emulsion-solvent evaporation process) coated with PEG and prostate-specific membrane antigen-targeting ligands and loaded with doxorubicin; currently BIND-014 is in clinical trials for the treatment of prostate and lung cancer ${ }^{8}$.

Micelles are composed of amphiphilic components that are organized by the hydrophobic effect to have a distinct lipophilic core and hydrophilic outer layer. In these systems, the drug cargo is typically limited to hydrophobic molecules entrapped in the core. GenexolPM is composed of a PEG-PLA block co-polymer loaded with paclitaxel, and has been investigated in clinical trials for breast, pancreatic, lung, and ovarian cancer ${ }^{106}$.

Dendrimers are branched tree-like structures which allow excellent control over size, dispersity, and functionalization during synthesis. Various types of drug cargo can be made to associate with the branched polymer matrix or attach to the dendrimer surface. SPL7013 (Vivagel ${ }^{\mathrm{TM}}$ ) is a lysine-based dendrimer with antimicrobial properties used for the prevention of HIV, genital herpes, and HPV ${ }^{107}$. 
Polyplexes are self-assembled nanoparticles that are stabilized by hydrophobic or electrostatic interactions with the constituent polymer(s) and drug cargo. CALAA-01 is a polyplex of PEGylated cyclodextrin loaded with siRNA (to knockdown the M2 subunit of ribonucleotide reductase) and coated with transferrin; CALAA-01 has been in clinical trials for the treatment of solid tumors ${ }^{9}$. Similarly, CRLX101 is a polyplex of PEGylated cyclodextrin conjugated to camptothecin that has been involved in clinical trials for renal, ovarian, and rectal cancer ${ }^{108}$.

As the field of nanoparticle delivery matures (Table 1), so must the principles guiding further innovation. Adopting a holistic view-which includes all aspects of nanocarrier design and deployment as inter-related tools to mediate targeting-will facilitate clinical translation of technologies and treatment paradigms. This integrated view may also shift the focus away from development of complex nanoparticle formulations- that are likely to be very difficult to scale for commercial availability - and towards simple, judiciously constructed solutions that are more likely to find clinical success. In this review we present a new outlook on targeting polymer nanoparticles for drug delivery, which we divide into anatomical, molecular, and temporal aspects (Figure 1). While our primary focus is on polymer nanoparticles, other drug delivery platforms, such as liposomes, are often employed under similar circumstances and therefore face many of the same challenges. As such, our conclusions present a broad perspective on targeting that will impact the design of all types of nanotherapeutics.

\section{CURRENT STATE OF THE ART IN NANOPARTICLE TARGETING}

The majority of current targeted nanoparticles are engineered to treat cancer by intravenous administration (Table 1); the details have been covered in several recent reviews ${ }^{4-6}$. Previous generations of tumor-targeted nanoparticles were designed to maximize passive targeting, in which systemically circulating nanoparticles penetrate the leaky vasculature often associated with tumors and accumulate due to slow clearance from poor lymphatic drainage (i.e. the enhanced permeability and retention, or EPR, effect $)^{7}$. In the passive targeting approach, increasing accumulation at the tumor correlates with longer circulation times and so the surface of these particles are coated with inert materials (such as polyethylene glycol or PEG) to reduce elimination of the particles via the host immune system and thereby maximize the circulation time. Seeking to further improve upon passive targeting, many current-generation nanoparticles rely on the promise of molecular (or active) targeting enhancements. In active targeting the surfaces of nanoparticles are endowed with molecules (e.g. native ligands or antibodies) that can, in theory, increase affinity for specific cells or tissues. Often active targeting is used in combination with nanoparticle formulations designed to have enhanced passive targeting. Two such polymer nanoparticle systems in clinical trials, BIND-014 and CALAA-01, are surface-modified with both PEG and targeting molecules that bind receptors enriched on some cancer cells ${ }^{8,9}$. While it is not the primary focus of this review, it is worth noting that there have also been some promising advances in the targeting of free drugs, such as the chemical stabilization of oligonucleotides ${ }^{10}$ and ligand-oligonucleotide conjugates ${ }^{11}$. 
Despite an abundance of attention on molecular approaches to targeting, the results have been mixed. While there are some intriguing successes, current technologies for ligandreceptor targeting of nanoparticles do not produce predictable outcomes ${ }^{12,13}$. There is growing evidence that the presence of targeting ligands can have a negative impact on passive targeting by reducing circulation times through enhanced immune elimination ${ }^{6,13}$. Additionally, the assumption that the specificity of a targeting ligand will be retained after conjugation to a nanoparticle surface is flawed, particularly when particles are delivered in $v_{i v o}{ }^{12}$. Furthermore, even if a highly specific targeted nanoparticle can be achieved, there is no guarantee that a unique receptor will be significantly expressed solely on the cell population of interest. These challenges have led to questions about whether the added complexity that comes with the introduction of active targeting ligands is valuable, particularly since complex nano-delivery systems are both difficult and expensive to develop to the point of clinical availability ${ }^{14}$. Thus, in spite of the promise arising from recent innovations in nanoparticle synthesis, it is unclear how much closer these innovations have gotten us to fulfilling the promise of drug targeting and where exactly the field should focus its attention moving forward.

We believe a more nuanced view of targeting will speed design of clinically useful systems to better deliver on the potential of nanoparticle drug delivery. A typical approach for design of targeted therapies focuses first on the development of a drug delivery platform, which is then screened against a variety of diseases to find the setting in which the greatest efficacy can be achieved. We prefer a "top-down" strategy in which a given disease indication fuels the design of nanomedicines. Recently, others have expressed a similar approach that starts by selecting a particular disease and then sampling relevant drug delivery platforms to identify the best method for treating that pathology ${ }^{15}$. Such an approach focuses attention on the biological characteristics that define a given disease, and how nanoparticles are likely to interact with cells and tissues in that context, and thus emphasizes design of a targeting system that is specifically tailored to these characteristics.

In addition to considering the disease state first, we argue that it is also essential to broaden the focus of nanoparticle delivery beyond the current emphasis on active targeting by intravenous delivery. For many diseases, alternative routes of administration may be more effective than systemic delivery. Further, regardless of whether targeting molecules are employed or not, it is critical to consider how the physicochemical properties of the nanoparticle and the surrounding biological milieu affect the ability to target at the molecular level. Finally, there are also important temporal aspects to targeting including the relationship between pharmacokinetics, drug release kinetics, and the therapeutic window for effective disease treatment. In the following sections, we expand upon this more comprehensive notion of targeting by providing examples of how a holistic approach can better fulfill the promise of targeted nanomedicines.

\section{ANATOMICAL TARGETING}

Targeting implies a direct focus on one object of interest, while simultaneously ignoring everything else. For nanoparticles that are intended to target a single cell population, this concept is difficult to translate into practice-particularly when the particles are 
administered systemically. While delivery to the blood circulation has the potential benefit of providing access to any vascularized tissue in the body, this pervasive access also increases the likelihood that unintended tissues will be targeted. It is typically observed that systemic delivery of nanoparticles results in significant accumulation in both liver and spleen as a result of immune clearance by the mononuclear phagocyte system (MPS; also known as reticuloendothelial system or RES) ${ }^{16}$. Unless these phagocytic cells of the liver (or spleen) are the intended target, this off-target accumulation provides a major impediment to achieving specificity with systemic delivery. Alternatively, directed local delivery of drugs is a simpler and (for some diseases) potentially more effective form of targeting, allowing a physical means for enrichment of nanoparticle concentration at a specific site and reduced accumulation in non-target tissues ${ }^{17-20}$. Local delivery has been shown useful in treatment of brain tumors; implantation of a macroscopic polymer wafer provides sustained effective concentrations of a chemotherapy drug in the brain, while sparing other tissues to drug exposure $^{21}$. Local administration can also be achieved with nanoparticles through a range of physical and minimally invasive targeting methods that are appropriate for specific diseases (Table 2). The following examples provide a sampling of how local modes of nanoparticle administration can be utilized as a means to enhance disease targeting.

\section{Brain infusion}

The most basic method for targeting by local administration of nanoparticles is direct injection into the tissue of interest. Such injections are invasive, but in some cases physiological barriers significantly impair the effectiveness of any other delivery route. The blood-brain barrier is one such physiological impediment where, despite decades of effort, no systemically targeted nanoparticle systems provide adequate transport across the BBB to allow effective treatment of serious brain diseases, such as glioblastoma multiforme ${ }^{17,22-25}$. Alternately, nanoparticles can be directly injected into the brain (circumventing the BBB) using catheters stereotactically targeted to precise anatomic locations; approaches such as convection-enhanced delivery (CED) can then be used to target larger volumes of the brain to ensure that the injected particles fully cover the targeted area $22,26,27$. Nanoparticles are potentially useful here because they allow for sustained intracellular delivery of the encapsulated agent. Sustained drug release is essential in this setting, because repeat dosing is not practical. In addition, effective delivery relies on the ability of nanoparticles to penetrate away from the site of injection and into proximal surrounding diseased tissue. For CED, nanoparticle size appears to be the main property that influences susceptibility to convective transport within the brain. For example, atypically small PLGA nanoparticles can be transported large distances from the site of infusion via convection ${ }^{23}$. The invasiveness of this delivery method will limit its broad application, but it is potentially useful for the delivery of agents that reverse Parkinson's disease ${ }^{28}$ or Huntington's disease ${ }^{29}$. In addition, this approach can be extended to other parts of the nervous system, as shown in the injection of protein growth factor-loaded nanoparticles for treatment of spinal cord injury ${ }^{30}$ or the injection of various nanoparticles into the eye for treatment of retinal diseases ${ }^{31,32}$.

\section{Dermal administration}

Direct local administration to target nanoparticles need not be as invasive as delivery to the brain. For example, topical application to the skin is non-invasive and yet still provides 
physical targeting. As evidence of the safety of this approach, chitosan, PLGA, poly(DLlactide) (PLA), polyalkylcyanoacrylate, and polycaprolactone (PCL) polymers are routinely used in dermal delivery for both therapeutic and cosmetic purposes ${ }^{18}$. Generally, topically applied nanoparticles are unable to deeply penetrate the stratum corneum (i.e. the outermost layer of the epidermis) and instead localize to proximal glands and hair follicles ${ }^{33}$. As such, topically applied nanoparticles typically cannot reach subepithelial capillaries to access the circulatory system. Nevertheless, particles can be formulated to penetrate deeply enough to have significant therapeutic benefits. For example, PLGA nanoparticles coated with a cationic lipid that induced swelling and opening of the stratum corneum were used to codeliver siRNA against TNF and capsacin (an anti-inflammatory drug) to treat the chronic inflammation of psoriasis ${ }^{18}$; importantly, this strategy has the potential to avoid the widespread immunosuppressive side-effects associated with systemic delivery of TNF antagonists ${ }^{34}$. The limited spread to surrounding tissues observed in dermal delivery provides a substantial safety benefit. In addition to this spatial advantage, the local retention of topically applied therapeutics imparts a temporal advantage by increasing treatment duration.

Both brain infusion and topical skin administration demonstrate the potential benefit of physically administering therapeutics directly at the intended site of action. However, local administration of nanoparticles via alternate routes does not always restrict targeting to the initial site of delivery. When subcutaneously injected, nanoparticles smaller than $100 \mathrm{~nm}$ are prone to clearance by lymphatic vessels, while larger nanoparticles are generally retained in the interstitial space near the site of injection ${ }^{35,36}$. As such, subcutaneous injection of sub-100 nm particles can be a method for targeted delivery to surrounding lymph nodes ${ }^{37-40}$, provided the particles are not so small as to leak into blood capillaries ${ }^{35}$. While targeting lymphatics by subcutaneous administration of sub- $100 \mathrm{~nm}$ nanoparticles can be inefficient ${ }^{41}$, this approach nevertheless has potential for the development of DNA-based vaccines $^{42}$ and in the treatment of certain lymphomas ${ }^{43}$.

\section{Mucosal delivery}

Mucosal administration offers the advantages of local tissue targeting ${ }^{44-46}$, while also providing potential routes for sustained systemic administration that are less invasive than intravenous injection ${ }^{47-49}$. Mucus provides a protective barrier on the epithelia of numerous tracts and structures in the body that are potential administration routes for nanotherapeutics. For example, topical vaginal administration of polymer nanoparticles allows for targeted delivery to the vaginal epithelium, and can be used to deliver siRNA ${ }^{50,51}$ and drugs ${ }^{52,53}$ in order to prevent and treat infectious diseases ${ }^{51,54}$ or cancer $^{55,56}$. Molecular engineering of these systems circumvents obstacles to local drug action; in particular, design of the nanoparticle surface allows particles to penetrate cervical mucus and to reach the underlying epithelium ${ }^{50,57,58}$. For example, addition of PEG to the nanoparticle surface enhances mucus penetration 58,59 , which can also be improved by modulating NP size and attraction to mucin fibres ${ }^{60,6162}$. Other surface elements, such as chitosan, can disrupt tight junctions and increase epithelial penetration ${ }^{63}$, providing a tool for controlling nanoparticle targeting after topical delivery to vagina, bladder, and other mucosal epithelial sites ${ }^{64,65}$. Alternately, another approach for mucosal delivery is to improve mucus adhesion ${ }^{66}$ or binding ${ }^{67}$, which 
then limits penetration to the epithelium, allows topical release of drug payloads, and links nanoparticle clearance to the natural clearance of mucus.

Regardless of the specific route of delivery, a consistent theme in the preceding examples is the need to carefully consider how the molecular properties of the delivered nanoparticlessuch as shape, size, charge, chemical character-will guide interactions with the local environment after delivery. Different aspects of targeting, even those that seemingly operate on very different scales (i.e. macroscopic versus microscopic), are nonetheless highly interdependent. Consequently, overcoming the obstacles associated with targeting requires moving beyond the optimization of individual elements of targeting, to instead focus on how all aspects work in concert. In the following sections, we introduce molecular aspects of targeted delivery and discuss specific issues that need to be addressed to ensure that molecular targeting strategies can be reliably incorporated within this holistic approach.

\section{Molecular targeting}

Given the potential for high affinity and specificity in conjugating a native ligand or relevant antibody to nanoparticles, it is understandable that so much attention in the field has been focused on these molecular targeting approaches. However, it is becoming clear that the highly specific targeting capabilities associated with molecules such as antibodies cannot simply be grafted on to the therapeutic functionality of a drug-loaded nanoparticle. The conjugation of targeting molecules to the surface of nanoparticles can not only impair the bioactivity of the targeting molecule ${ }^{12}$, but also negatively impact the therapeutic efficacy of a nanoparticle by reducing passive targeting via enhanced immune elimination ${ }^{13}$. Moreover, it has been demonstrated that the addition of targeting ligands does not significantly alter the biodistribution of systemically administered nanoparticles ${ }^{5,68,69}$. In spite of these challenges, active targeting can still play a role as part of the broader toolkit of targeted drug delivery, particularly when the route of administration ensures that the conjugated ligand will find its intended receptor. However, issues that can impact both specificity and therapeutic efficacy must be addressed to achieve success with molecular targeting strategies (Table 3). The following sections highlight some of the most significant challenges.

\section{Targeting specificity challenges}

Most polymer nanoparticle formulations are taken up —at least to some extent—by many different types of cells, even in the absence of modifications intended to enhance nanoparticle internalization ${ }^{70,71}$. While an inherent propensity for cellular uptake can be therapeutically beneficial, it nevertheless provides an impediment to specific targeting by reducing the ability to regulate unintended particle uptake. Cellular uptake can be sensitive to physical characteristics of nanoparticles-properties such as particle shape, size, charge, and hydrophobicity ${ }^{71-75}$ - thereby providing a potential means for controlling the level of uptake in the absence of a specific targeting ligand. However, without a singular mechanism linking particle properties to cellular uptake, it is difficult to predict what combination of properties will be most relevant for a given nanoparticle formulation and cellular target. Moreover, uptake observed for one cell in one environment may not occur for all cells in all environments, making it risky to extrapolate from in vitro mono-cell culture models to in vivo conditions. These caveats aside, it has been shown both in vivo and in vitro that coating 
the surface of polymeric nanoparticles with inert molecules such as $\mathrm{PEG}^{76}$ or hyperbranched polyglycerol ${ }^{77}$ can significantly reduce unwanted cellular interactions.

Many studies have focused on understanding the basic mechanisms underlying this intrinsic capacity of nanoparticles to be taken up by cells ${ }^{71}$. In some cases this non-specific particle uptake may be attributable to electrostatic properties of the particles; for example, cationic particles associate on the basis of ionic interactions with anionic cell membranes and cell surface components leading to enhanced particle uptake ${ }^{78}$. But perhaps more importantly (especially in vivo), there is now substantial evidence that surface adsorbed serum or plasma proteins are critical determinants of cell-nanoparticle interactions ${ }^{12,79,80}$. These adsorbed proteins, often referred to as a protein corona, can rapidly (within seconds) associate with nanoparticles upon exposure in serum containing medium or blood. Using a mass spectrometry based approach, polystyrene nanoparticles were shown to associate with hundreds of different serum proteins; the specific proteins adsorbed depended on particle charge, size, and chemical composition ${ }^{79}$. Because of this, the ability of surface-conjugated molecules (such as transferrin) to provide specific nanoparticle targeting can be stifled by the presence of serum proteins ${ }^{12}$. Moreover, many of the serum proteins that adsorb to nanoparticle surfaces may themselves activate receptor-mediated endocytosis ${ }^{16}$. Consequently, what is observed as a non-specific effect may actually result from an unintended, specific interaction.

Thus - whether by inhibiting the biological activity of a targeting ligand or by providing alternative, unintended routes of cellular uptake-proteins adsorbed from the environment can interfere with active targeting. This role of serum proteins on drug targeting extends beyond the field of nanoparticles: binding to albumin can prevent renal filtration of macromolecular drugs ${ }^{81}$ and certain lipoproteins have been observed to direct the tissue accumulation and cellular uptake of various nucleic acid therapeutics ${ }^{82}$. These other fields have adapted to exploit drug interactions with serum components. Similarly, better means for either controlling the composition of the nanoparticle protein corona or minimizing its effects must be developed ${ }^{80,83}$. Such strategies should focus not only on reducing uptake by phagocytic cells of the immune system (as is often the motivation with surface PEGylation), but also on preserving targeting specificity. Finally, it will be important to better understand how the protein corona varies with the physicochemical properties of a given particle, and with different anatomical routes of administration ${ }^{80}$.

The ability to generate specific antibodies against any cell surface receptor would appear to promise a wide palette of potential targets from which to choose. However, the vast majority of these molecules are not unique to a single cell population, which severely restricts the number of surface receptors useful for targeting purposes. As a result, the majority of studies have focused on just a few specific targets typically overexpressed on cancer cells (e.g. transferrin receptor and HER2) $)^{5}$. Expanding the list of potential targets for a given pathology necessarily requires understanding the fundamental biology underlying the disease. It also requires understanding from a biochemical perspective the surface expression required on the targeted cell relative to non-targeted cells and nanoparticle avidity in order to ensure strong nanoparticle association. In instances where a suitable cell surface target does exist, it is also important to realize that the binding of the targeting 
moiety to the targeted receptor may itself lead to a biologic response that can either enhance or inhibit the desired therapeutic effect ${ }^{84}$.

\section{Therapeutic efficacy challenges}

Even if a nanoparticle can successfully navigate these pitfalls, and be specifically taken up in a targeted cell, this does not guarantee therapeutic efficacy. The intracellular fate of nanoparticles, and the timing of agent release, determines whether the active agent will find its intended site of action. A canonical example of this challenge is in the delivery of siRNA to the cytosol ${ }^{85}$. The therapeutic potential of siRNA is far-reaching, with the possibility to impact diverse disease states (such as chronic inflammatory disorders, neurodegenerative disorders, viral infections, organ transplant rejection, and cancer ${ }^{86}$ ) with high target specificity. In order to be effective, siRNA molecules need to reach the endogenous processing machinery in the cytosol, a difficult challenge given the instability and short halflife of extracellular siRNA. Various nanoparticle formulations have been engineered to successfully protect siRNA from degradation, but many of these formulations become trapped in endosomal compartments and/or trafficked to lysosomes where the particle and siRNA are ultimately degraded without ever reaching their target ${ }^{87}$. To combat this, a great deal of effort has been focused on facilitating endosomal escape by adding drugs ${ }^{88}$, cationic polymers $^{89}$, lipids ${ }^{90}$, or fusogenic peptides ${ }^{91}$ to the nanoparticle formulation.

Enhancing siRNA delivery via endosome escape is just one example of controlling nanoparticle fate in cells; there is a diverse array of molecular modifications that can aid in regulating intracellular delivery and localization ${ }^{92}$. For example, cell-penetrating peptides (CPPs) are a widely used nanoparticle modification to improve cell uptake and modulate intracellular fate; however, the functional mechanisms of many CPPs (let alone those attached to nano-scale structures) are still unclear ${ }^{93}$. Gaps in the understanding between activity and mechanism (as well as potential for toxicity) suggest caution in the use of molecular modifications, providing another illustration of how each aspect of molecular composition must be considered as an element in a holistic design.

Achieving cellular specificity through active targeting remains the (non-trivial) goal of many nanomedicines. Active targeting is confounded by various factors including nanoparticle physicochemical properties, interactions specific to route of delivery, and challenges unique to the agent being delivered, e.g. cytosolic delivery of siRNA. To further complicate this issue, some efficacious actively targeted nanoparticles possess only a narrow window over which the targeting capacity of a conjugated ligand will be retained ${ }^{94}$. Consequently, it cannot be assumed that any given disease or nanoparticle platform will be compatible with active targeting approaches. This fact reinforces the argument that active molecular targeting should not be viewed as the only approach for targeting disease. Rather, ligand conjugation is appropriate only when: 1) the route of delivery can get the nanoparticle to the cell displaying the targeted receptor of interest without compromising the therapeutic efficacy of the delivered drug; and 2) the intracellular fate of the nanoparticle after uptake can support effective therapeutic delivery. These requirements will not be present in all clinical settings, or for all agents. By biasing the use of molecular targeting strategies strictly to diseases and 
treatment modalities that meet these criteria, we will significantly improve the odds of successfully incorporating molecular targeting in therapeutic design.

\section{TEMPORAL}

In addition to selection of administration route and controlling molecular interactions, nanoparticle targeting can be further improved with a temporal perspective. Temporal targeting exploits therapeutic windows to maximize drug delivery. For example, lungs have a larger window of therapeutic opportunity for systemically administered agents than spleen; the respective times required for lungs and spleen to receive a volume equivalent to the total blood volume in humans are $\sim 1$ and 64 minutes ${ }^{95}$. In the context of nanoparticle therapeutics, temporal targeting can be achieved through an understanding of disease progression and pharmacokinetics, as well as through the engineering of nanoparticles that impart control over when a drug is delivered.

\section{Biological timing}

Disease pathophysiology can significantly impair or improve the amenability to nanoparticle treatment. Developing targeted nanomedicines with a disease-first approach requires a detailed understanding of pathophysiology, since treatment susceptibility for diseases often lessens with time. Typically, treating disease at early stages will prevent further spread and pathogenesis, but early treatment may also be advantageous from a pharmacokinetic perspective. For example, in cancer, elevated tumor interstitial fluid pressure (IFP) results from a combination of poorly formed vasculature and lack of functional lymphatics, which in turn, reduces convective flow of nanoparticles into the tumor parenchyma ${ }^{96}$. As tumors grow, IFP typically intensifies and further impedes nanoparticle transport ${ }^{97}$. Thus, tumor delivery of nanoparticles can be improved through temporal targeting of early-stage tumors (Figure 2a). Similarly, cystic fibrosis involves thickening of mucus lining the airways and intestinal tract, which can exacerbate the mucosal barrier and impede delivery of nanotherapeutics ${ }^{98}$. Although nanoparticles with muco-adhesive and muco-penetrating properties have been engineered to exploit and overcome mucus retention ${ }^{62,67,99,100}$, early treatment of cystic fibrosis may circumvent this obstacle (Figure 2b). Note that in addition to the temporal aspects of disease pathology, other aspects of biological timing can have an impact on therapeutic windows. For example, the cycling of the female reproductive mucosa can impact the timing for administration of intravaginal or intrauterine therapies. Likewise, the cycle of expansion and contraction of the bladder influences tissue structure in the bladder wall and therefore likely influences tissue permeability to nanoparticles as well.

Designing nanomedicines that target specific therapeutic windows for a given disease may be limited in some cases by an incomplete understanding of disease pathogenesis. Preclinical models can be predictive, but not necessarily representative of disease progression in humans. For example, the EPR effect observed in many pre-clinical models may be less prevalent in human tumors ${ }^{101}$. A key benefit of nanoparticle platforms is their amenability to deliver a diverse array of cargos. Thus multifunctional nanoparticles can combine therapy with imaging modalities (i.e. theranostics ${ }^{102}$ ) in order to help identify times when a given disease is most susceptible to treatment. A common example is to fabricate nanoparticles loaded with a chemotherapeutic and functionalized with an imaging contrast agent ${ }^{103}$. 
However, similar to the concerns with molecular targeting, the added complexity of theranostic nanosystems suggests a careful approach.

\section{Controlled release}

In addition to the capacity for multifunctional properties, another property of polymer nanoparticles is the ability to protect drug cargo from the time of administration to delivery at the intended tissue, cell, or intracellular location, and to release it in a sustained fashion. Given the numerous physiological and cellular barriers facing the delivery of nanotherapeutics, control over when to release an encapsulated drug can significantly impact therapeutic efficacy. Unlike other classes of nanocarriers, most polymer nanoparticle systems can be tuned to yield desirable release kinetics. This process, known as controlled release, is typically achieved by regulating the rates of polymer biodegradation and drug diffusion outward through the polymer matrix. As such, polymer nanosystems have been developed with release durations ranging from minutes to weeks ${ }^{104}$; in particular, biodegradable poly(ester) polymers such as PLA and PLGA are well known for use in formulating controlled release nanoparticles. A primary goal in developing these systems is to align drug release profiles with nanoparticle pharmacokinetics. For example, the drug release profiles of various PLA nanoparticles (with similar composition to BIND-014) were tuned to release over several days in order to capitalize on the extended systemic circulation time of the PEGylated nanocarriers, which ultimately accumulated in tumors ${ }^{8}$. In another example, PLGA nanoparticles administered intranasally to a model of cystic fibrosis resided within diseased lungs and achieved pharmacological effects through sustained drug release for up to 11 days ${ }^{19}$.

Another approach to controlling drug release is the development of modular nanoparticles in which delivery is triggered. For example, a polymer nanoparticle system was designed to sequentially deliver an anti-angiogenesis agent followed by a chemotherapy agent ${ }^{105}$. This approach triggered vascular shutdown and entrapped the nanoparticles within a tumor before releasing chemotherapy; thereby temporally targeting drug release to coincide with the time at which a disease was susceptible to treatment. With the ability to respond when needed, modular nanosystems may be paradoxically simpler than some complex molecularly targeted nanoparticles. Through the coordinated alignment of pharmacokinetics and drug delivery with therapeutic windows provided by disease pathophysiology, polymer nanoparticles present a unique technology for temporal targeting of disease, which has not yet been fully exploited.

\section{CONCLUSION}

Polymer nanoparticles can be synthesized with control over particle composition, incorporation of many kinds of drug agents with tunable release kinetics, and presentation of targeting ligands. But the effective use of these nanomaterials for targeted treatment of human disease is still limited. We believe that effective targeting requires thinking along several length scales - from macroscopic to microscopic. Here, we present a holistic approach to nanoparticle design, which benefits from the enormous progress that has been made over the past decade. This new approach-encompassing the route of administration, 
molecular composition of the nanocarrier, and temporal coordination-requires that nanoparticle design be directed by pathophysiology and be integrated over all of these elements. This holistic perspective has already been applied in some settings; for example, the aforementioned local delivery of small nanoparticles for controlled drug release in the brain engages all of these targeting elements. Additionally, these principles are not mutually exclusive. For example, molecular modifications and drug release kinetics should be tuned for a given nanoparticle administration route. We suggest that deliberate application of this holistic approach will substantially reduce the hurdles in developing polymer nanoparticles for effective treatment of cancer and many other difficult to manage diseases. In fact, the holistic view we have outlined here is not fully inclusive; in the emerging era of personalized medicine, drugs themselves can impart another layer of targeting. Ultimately, the definition of different aspects of targeting does not matter as much as the approach taken to achieve targeting, which we believe should consider every possible tool in the arsenal in order to achieve the desired therapeutic end.

\section{GLOSSARY}

Active targeting

Enhanced
permeability and
retention (EPR)
effect

Passive targeting

Targeting
Targeting that is mediated by specific receptor-ligand interactions. For active targeting with nanoparticles, ligands are usually attached to the particle surface to enhance cell- or tissue-specific binding and nanoparticle uptake through receptor-mediated endocytosis

Accumulation of particles in tumors due to extravasation from the blood through leaky vasculature (enhanced permeability) and lack of lymphatic drainage (retention). Nanoparticles of diameter near 100 $\mathrm{nm}$ appear to be optimal for the EPR effect in many tumors

Targeting that occurs due to physical properties of a nanoparticle, such as surface charge or size, that decrease protein opsonization and phagocytic elimination, enhancing circulation time and subsequently retention in tumors

The preferential accumulation of nanoparticles in a preferred (or target site) when compared to other (non-target) sites

\section{References}

1. Liu XM, et al. Syntheses of click PEG-dexamethasone conjugates for the treatment of rheumatoid arthritis. Biomacromolecules. 2010; 11:2621-8. [PubMed: 20831200]

2. Wong C, et al. Multistage nanoparticle delivery system for deep penetration into tumor tissue. Proc Natl Acad Sci U S A. 2011; 108:2426-31. [PubMed: 21245339]

3. Kamaly N, Xiao Z, Valencia PM, Radovic-Moreno AF, Farokhzad OC. Targeted polymeric therapeutic nanoparticles: design, development and clinical translation. Chem Soc Rev. 2012; 41:2971-3010. [PubMed: 22388185]

4. Shi J, Xiao Z, Kamaly N, Farokhzad OC. Self-Assembled Targeted Nanoparticles: Evolution of Technologies and Bench to Bedside Translation. Accounts of Chemical Research. 2011; 44:11231134. [PubMed: 21692448]

5. Bertrand N, Wu J, Xu X, Kamaly N, Farokhzad OC. Cancer nanotechnology: the impact of passive and active targeting in the era of modern cancer biology. Adv Drug Deliv Rev. 2014; 66:2-25. [PubMed: 24270007] 
6. Lammers T, Kiessling F, Hennink WE, Storm G. Drug targeting to tumors: principles, pitfalls and (pre-) clinical progress. J Control Release. 2012; 161:175-87. [PubMed: 21945285]

7. Iyer AK, Khaled G, Fang J, Maeda H. Exploiting the enhanced permeability and retention effect for tumor targeting. Drug Discov Today. 2006; 11:812-8. [PubMed: 16935749]

8. Hrkach J, et al. Preclinical development and clinical translation of a PSMA-targeted docetaxel nanoparticle with a differentiated pharmacological profile. Sci Transl Med. 2012; 4:128ra39.

9. Davis ME, et al. Evidence of RNAi in humans from systemically administered siRNA via targeted nanoparticles. Nature. 2010; 464:1067-70. [PubMed: 20305636]

10. Merki E, et al. Antisense oligonucleotide directed to human apolipoprotein B-100 reduces lipoprotein(a) levels and oxidized phospholipids on human apolipoprotein B-100 particles in lipoprotein(a) transgenic mice. Circulation. 2008; 118:743-53. [PubMed: 18663084]

11. Cheng CJBR, Babar IA, Pincus Z, Barrera F, Liu C, Svoronos A, Braddock DT, Glazer PM, Engelman DM, Saltzman WM, Slack FJ. MicroRNA silencing for cancer therapy targeted to the tumor microenvironment. Nature. in press.

12. Salvati A, et al. Transferrin-functionalized nanoparticles lose their targeting capabilities when a biomolecule corona adsorbs on the surface. Nat Nanotechnol. 2013; 8:137-43. [PubMed: 23334168]

13. Kunjachan $\mathrm{S}$, et al. Passive versus active tumor targeting using RGD- and NGR-modified polymeric nanomedicines. Nano Lett. 2014; 14:972-81. [PubMed: 24422585]

14. Cheng Z, Al Zaki A, Hui JZ, Muzykantov VR, Tsourkas A. Multifunctional nanoparticles: cost versus benefit of adding targeting and imaging capabilities. Science. 2012; 338:903-10. [PubMed: 23161990]

15. Crielaard BJ, Lammers T, Schiffelers RM, Storm G. Drug targeting systems for inflammatory disease: one for all, all for one. J Control Release. 2012; 161:225-34. [PubMed: 22226771]

16. Bertrand N, Leroux JC. The journey of a drug-carrier in the body: an anatomo-physiological perspective. J Control Release. 2012; 161:152-63. [PubMed: 22001607]

17. Bernal GM, et al. Convection-enhanced delivery and in vivo imaging of polymeric nanoparticles for the treatment of malignant glioma. Nanomedicine. 2014; 10:149-57. [PubMed: 23891990]

18. Desai PR, et al. Topical delivery of anti-TNFa siRNA and capsaicin via novel lipid-polymer hybrid nanoparticles efficiently inhibits skin inflammation in vivo. Journal of controlled release: official journal of the Controlled Release Society. 2013; 170:51-63. [PubMed: 23643662]

19. Vij N, et al. Development of PEGylated PLGA nanoparticle for controlled and sustained drug delivery in cystic fibrosis. Journal of Nanobiotechnology. 2010; 8

20. Weiser JR, Saltzman WM. Controlled release for local delivery of drugs: barriers and models. J Control Release. 2014

21. Fleming AB, Saltzman WM. Pharmacokinetics of the carmustine implant. Clinical Pharmacokinetics. 2002; 41:403-419. [PubMed: 12074689]

22. Sawyer AJ, Saucier-Sawyer JK, Booth CJ, Liu J, Patel T, Piepmeier JM, Saltzman WM. Convection-enhanced delivery of camptothecin-loaded polymer nanoparticles for treatmetn of intracranial tumors. Drug delivery and Translational Research. 2011:34-42. [PubMed: 21691426]

23. Zhou J, et al. Highly penetrative, drug-loaded nanocarriers improve treatment of glioblastoma. Proc Natl Acad Sci U S A. 2013; 110:11751-6. [PubMed: 23818631]

24. Nance EA, et al. A dense poly(ethylene glycol) coating improves penetration of large polymeric nanoparticles within brain tissue. Sci Transl Med. 2012; 4:149ra119.

25. Neeves KB, Sawyer AJ, Foley CP, Saltzman WM, Olbricht WL. Dilation and degradation of the brain extracellular matrix enhances penetration of infused polymer nanoparticles. Brain Res. 2007; 1180:121-32. [PubMed: 17920047]

26. Sawyer AJ, Piepmeier JM, Saltzman WM. New methods for direct delivery of chemotherapy for treating brain tumors. Yale J Biol Med. 2006; 79:141-52. [PubMed: 17940624]

27. Allard E, Passirani C, Benoit JP. Convection-enhanced delivery of nanocarriers for the treatment of brain tumors. Biomaterials. 2009; 30:2302-18. [PubMed: 19168213]

28. Lam MF, Thomas Mg, Lind CRP. Neurosurgical convection-enhanced delivery of treatments for Parkinson's disease. 
29. Stiles DK, et al. Widespread suppression of huntingtin with convection-enhanced delivery of siRNA.

30. Wang YC, et al. Sustained intraspinal delivery of neurotrophic factor encapsulated in biodegradable nanoparticles following contusive spinal cord injury. Biomaterials. 2008; 29:454653. [PubMed: 18774604]

31. Li H, et al. A PEDF N-terminal peptide protects the retina from ischemic injury when delivered in PLGA nanospheres. Exp Eye Res. 2006; 83:824-33. [PubMed: 16822505]

32. Kaur IP, Kakkar S. Nanotherapy for posterior eye diseases. J Control Release. 2014

33. Baroli B. Penetration of nanoparticles and nanomaterials in the skin: fiction or reality? Journal of pharmaceutical sciences. 2010; 99:21-50. [PubMed: 19670463]

34. Hadam J, Aoun E, Clarke K, Wasko MC. Managing risks of TNF inhibitors: an update for the internist. Cleve Clin J Med. 2014; 81:115-27. [PubMed: 24493494]

35. Moghimi SM, Bonnemain B. Subcutaneous and intravenous delivery of diagnostic agents to the lymphatic system: applications in lymphoscintigraphy and indirect lymphography. Advanced Drug Delivery Reviews. 1999; 37:295-312. [PubMed: 10837741]

36. Irvine DJ, Swartz MA, Szeto GL. Engineering synthetic vaccines using cues from natural immunity. Nature Materials. 2013; 12:978-990.

37. Moghimi SM, Rajabi-Siahboomi AR. Advanced colloid-based systems for efficient delivery of drugs and diagnostic agents to the lymphatic tissues. Progress in Biophysics and Molecular Biology. 1996; 65:221-249. [PubMed: 9062433]

38. Hawley AE, Illum L, Davis SS. Lymph node localisation of biodegradable nanospheres surface modified with poloxamer and poloxamine block co-polymers. FEBS Letters. 1997; 400:319-323. [PubMed: 9009222]

39. Kreuter J, Nefzger M, Liehl E, Czok R, Voges R. Distribution and elimination of poly(methyl methacrylate) nanoparticles after subcutaneous administration to rats. Journal of Pharmaceutical Sciences. 1983; 72:1146-1149. [PubMed: 6644560]

40. Toita R, et al. Biodistribution of 125I-labeled polymeric vaccine carriers after subcutaneous injection. Bioorganic \& Medicinal Chemistry. 2013; 21:5310-5315. [PubMed: 23830700]

41. Moghimi SM, et al. Surface engineered nanospheres with enhanced drainage into lymphatics and uptake by macrophages of the regional lymph nodes. FEBS Letters. 1994; 344:25-30. [PubMed: 8181558]

42. DeMuth PC, et al. Polymer multilayer tattooing for enhanced DNA vaccination. Nat Mater. 2013; 12:367-76. [PubMed: 23353628]

43. Reddy LH, Sharma RK, Murthy RSR. Enhanced tumour uptake of doxorubicin loaded poly(butyl cyanoacrylate) nanoparticles in mice bearing Dalton's lymphoma tumour. Journal of Drug Targeting. 2004; 12:443-451. [PubMed: 15621669]

44. Cartiera MS, et al. Partial correction of cystic fibrosis defects with PLGA nanoparticles encapsulating curcumin. Mol Pharm. 2010; 7:86-93. [PubMed: 19886674]

45. Gugulothu D, Kulkarni A, Patravale V, Dandekar P. pH-sensitive nanoparticles of curcumincelecoxib combination: evaluating drug synergy in ulcerative colitis model. J Pharm Sci. 2014; 103:687-96. [PubMed: 24375287]

46. Yoo MK, et al. Targeted delivery of chitosan nanoparticles to Peyer's patch using M cell-homing peptide selected by phage display technique. Biomaterials. 2010; 31:7738-47. [PubMed: 20656343]

47. Gundogdu E, Yurdasiper A. Drug Transport Mechanism of Oral Antidiabetic Nanomedicines. Int J Endocrinol Metab. 2014; 12:e8984. [PubMed: 24696697]

48. Pridgen EM, et al. Transepithelial transport of Fc-targeted nanoparticles by the neonatal fc receptor for oral delivery. Sci Transl Med. 2013; 5:213ra167.

49. Mohammad AK, Amayreh LK, Mazzara JM, Reineke JJ. Rapid Lymph Accumulation of Polystyrene Nanoparticles Following Pulmonary Administration. Pharmaceutical Research. 2013; 30:424-434. [PubMed: 22992832]

50. Woodrow KA, et al. Intravaginal gene silencing using biodegradable polymer nanoparticles densely loaded with small-interfering RNA. Nat Mater. 2009; 8:526-33. [PubMed: 19404239] 
51. Steinbach JM, Weller CE, Booth CJ, Saltzman WM. Polymer nanoparticles encapsulating siRNA for treatment of HSV-2 genital infection. J Control Release. 2012; 162:102-10. [PubMed: 22705461]

52. Lai SK, Wang YY, Hanes J. Mucus-penetrating nanoparticles for drug and gene delivery to mucosal tissues. Adv Drug Deliv Rev. 2009; 61:158-71. [PubMed: 19133304]

53. Ensign LM, et al. Mucus-penetrating nanoparticles for vaginal drug delivery protect against herpes simplex virus. Sci Transl Med. 2012; 4:138ra79.

54. Navath RS, et al. Injectable PAMAM dendrimer-PEG hydrogels for the treatment of genital infections: formulation and in vitro and in vivo evaluation. Mol Pharm. 2011; 8:1209-23. [PubMed: 21615144]

55. Yang M, et al. Vaginal Delivery of Paclitaxel via Nanoparticles with Non-Mucoadhesive Surfaces Suppresses Cervical Tumor Growth. Adv Healthc Mater. 2013

56. Blum JS, WC, Booth CJ, Babar IA, Liang X, Slack FJ, Saltzman WM. Topical treatment of K-ras and Pten mediated intravaginal cancer with camptothecin-loaded nanoparticles. Drug Delivery and Translational Research. 2011; 1:383-394. [PubMed: 25419505]

57. das Neves J, et al. Biodistribution and Pharmacokinetics of Dapivirine-Loaded Nanoparticles after Vaginal Delivery in Mice. Pharm Res. 2014

58. Cu Y, Booth CJ, Saltzman WM. In vivo distribution of surface-modified PLGA nanoparticles following intravaginal delivery. J Control Release. 2011; 156:258-64. [PubMed: 21763739]

59. Lai SK, et al. Rapid transport of large polymeric nanoparticles in fresh undiluted human mucus. Proc Natl Acad Sci U S A. 2007; 104:1482-7. [PubMed: 17244708]

60. das Neves J, Amiji M, Sarmento B. Mucoadhesive nanosystems for vaginal microbicide development: friend or foe? Wiley Interdiscip Rev Nanomed Nanobiotechnol. 2011; 3:389-99. [PubMed: 21506290]

61. Cu Y, Saltzman WM. Drug delivery: Stealth particles give mucus the slip. Nat Mater. 2009; 8:113. [PubMed: 19096387]

62. Cu Y, Saltzman WM. Controlled surface modification with poly(ethylene)glycol enhances diffusion of PLGA nanoparticles in human cervical mucus. Mol Pharm. 2009; 6:173-81. [PubMed: 19053536]

63. GuhaSarkar S, Banerjee R. Intravesical drug delivery: Challenges, current status, opportunities and novel strategies. J Control Release. 2010; 148:147-59. [PubMed: 20831887]

64. Barthelmes J, Perera G, Hombach J, Dunnhaupt S, Bernkop-Schnurch A. Development of a mucoadhesive nanoparticulate drug delivery system for a targeted drug release in the bladder. Int $\mathrm{J}$ Pharm. 2011; 416:339-45. [PubMed: 21726619]

65. Martin DT, et al. Surface-modified nanoparticles enhance transurothelial penetration and delivery of survivin siRNA in treating bladder cancer. Mol Cancer Ther. 2014; 13:71-81. [PubMed: 24222663]

66. Peppas NA, Huang Y. Nanoscale technology of mucoadhesive interactions. Adv Drug Deliv Rev. 2004; 56:1675-87. [PubMed: 15350296]

67. Wang YY, et al. Mucoadhesive nanoparticles may disrupt the protective human mucus barrier by altering its microstructure. PLoS One. 2011; 6:e21547. [PubMed: 21738703]

68. Kirpotin DB, et al. Antibody targeting of long-circulating lipidic nanoparticles does not increase tumor localization but does increase internalization in animal models. Cancer Res. 2006; 66:673240. [PubMed: 16818648]

69. Pirollo KF, Chang EH. Does a targeting ligand influence nanoparticle tumor localization or uptake? Trends Biotechnol. 2008; 26:552-8. [PubMed: 18722682]

70. He C, Hu Y, Yin L, Tang C, Yin C. Effects of particle size and surface charge on cellular uptake and biodistribution of polymeric nanoparticles. Biomaterials. 2010; 31:3657-66. [PubMed: 20138662]

71. Sahay G, Alakhova DY, Kabanov AV. Endocytosis of nanomedicines. J Control Release. 2010; 145:182-95. [PubMed: 20226220]

72. Loverde SM, Klein ML, Discher DE. Nanoparticle shape improves delivery: rational coarse grain molecular dynamics (rCG-MD) of taxol in worm-like PEG-PCL micelles. Adv Mater. 2012; 24:3823-30. [PubMed: 22105885] 
73. Perry JL, Herlihy KP, Napier ME, Desimone JM. PRINT: a novel platform toward shape and size specific nanoparticle theranostics. Acc Chem Res. 2011; 44:990-8. [PubMed: 21809808]

74. Verma A, Stellacci F. Effect of surface properties on nanoparticle-cell interactions. Small. 2010; 6:12-21. [PubMed: 19844908]

75. Kolhar P, et al. Using shape effects to target antibody-coated nanoparticles to lung and brain endothelium. Proc Natl Acad Sci U S A. 2013; 110:10753-8. [PubMed: 23754411]

76. Perry JL, et al. PEGylated PRINT nanoparticles: the impact of PEG density on protein binding, macrophage association, biodistribution, and pharmacokinetics. Nano Lett. 2012; 12:5304-10. [PubMed: 22920324]

77. Deng Y, et al. The effect of hyperbranched polyglycerol coatings on drug delivery using degradable polymer nanoparticles. Biomaterials. 2014; 35:6595-602. [PubMed: 24816286]

78. Huhn D, et al. Polymer-coated nanoparticles interacting with proteins and cells: focusing on the sign of the net charge. ACS Nano. 2013; 7:3253-63. [PubMed: 23566380]

79. Tenzer $S$, et al. Rapid formation of plasma protein corona critically affects nanoparticle pathophysiology. Nat Nanotechnol. 2013; 8:772-81. [PubMed: 24056901]

80. Monopoli MP, Aberg C, Salvati A, Dawson KA. Biomolecular coronas provide the biological identity of nanosized materials. Nat Nanotechnol. 2012; 7:779-86. [PubMed: 23212421]

81. Crooke ST, et al. Pharmacokinetic properties of several novel oligonucleotide analogs in mice. The Journal of Pharmacology and Experimental Therapeutics. 1996; 277:923-937. [PubMed: 8627575]

82. Wolfrum C, et al. Mechanisms and optimization of in vivo delivery of lipophilic siRNAs. Nature Biotechnology. 2007; 25:1149-1157.

83. Rodriguez PL, et al. Minimal "Self” peptides that inhibit phagocytic clearance and enhance delivery of nanoparticles. Science. 2013; 339:971-5. [PubMed: 23430657]

84. Panariti A, Miserocchi G, Rivolta I. The effect of nanoparticle uptake on cellular behavior: disrupting or enabling functions? Nanotechnol Sci Appl. 2012; 5:87-100. [PubMed: 24198499]

85. Wang Y, Huang L. A window onto siRNA delivery. Nat Biotechnol. 2013; 31:611-2. [PubMed: 23839146]

86. Bumcrot D, Manoharan M, Koteliansky V, Sah DW. RNAi therapeutics: a potential new class of pharmaceutical drugs. Nat Chem Biol. 2006; 2:711-9. [PubMed: 17108989]

87. Dominska M, Dykxhoorn DM. Breaking down the barriers: siRNA delivery and endosome escape. J Cell Sci. 2010; 123:1183-9. [PubMed: 20356929]

88. Zhou J, Patel TR, Fu M, Bertram JP, Saltzman WM. Octa-functional PLGA nanoparticles for targeted and efficient siRNA delivery to tumors. Biomaterials. 2012; 33:583-91. [PubMed: 22014944]

89. Lee JS, et al. Gold, poly(beta-amino ester) nanoparticles for small interfering RNA delivery. Nano Lett. 2009; 9:2402-6. [PubMed: 19422265]

90. Dahlman JE, et al. In vivo endothelial siRNA delivery using polymeric nanoparticles with low molecular weight. Nat Nanotechnol. 2014

91. Cheng CJ, Saltzman WM. Enhanced siRNA delivery into cells by exploiting the synergy between targeting ligands and cell-penetrating peptides. Biomaterials. 2011; 32:6194-203. [PubMed: 21664689]

92. Biswas S, Torchilin VP. Nanopreparations for organelle-specific delivery in cancer. Advanced Drug Delivery Reviews. 2014; 66:26-41. [PubMed: 24270008]

93. Madani F, et al. Mechanisms of Cellular Uptake of Cell-Penetrating Peptides. Journal of Biophysics. 2011; 2011

94. Schaffer DV, Lauffenburger DA. Optimization of cell surface binding enhances efficiency and specificity of molecular conjugate gene delivery. J Biol Chem. 1998; 273:28004-9. [PubMed: 9774415]

95. Bertrand N, Leroux JC. The journey of a drug-carrier in the body: an anatomo-physiological perspective. Journal of Controlled Release: Official Journal of the Controlled Release Society. 2012; 161:152-163. [PubMed: 22001607] 
96. Boucher Y, Baxter Lt, Jain RK. Interstitial pressure gradients in tissue-isolated and subcutaneous tumors: implications for therapy.

97. Torosean S, et al. Nanoparticle uptake in tumors is mediated by the interplay of vascular and collagen density with interstitial pressure. Nanomedicine: Nanotechnology, Biology, and Medicine. 2013; 9:151-158.

98. Boucher RC. An overview of the pathogenesis of cystic fibrosis lung disease. Advanced Drug Delivery Reviews. 2002; 54:1359-1371. [PubMed: 12458149]

99. Suk JS, et al. Rapid transport of muco-inert nanoparticles in cystic fibrosis sputum treated with Nacetyl cysteine. Nanomedicine (Lond). 2011; 6:365-75. [PubMed: 21385138]

100. Yang M, et al. Biodegradable nanoparticles composed entirely of safe materials that rapidly penetrate human mucus. Angew Chem Int Ed Engl. 2011; 50:2597-600. [PubMed: 21370345]

101. Prabhakar U, et al. Challenges and Key Considerations of the Enhanced Permeability and Retention Effect for Nanomedicine Drug Delivery in Oncology. Cancer Research. 2013; 73:2412-2417. [PubMed: 23423979]

102. Kelkar SS, Reineke TM. Theranostics: Combining Imaging and Therapy. Bioconjugate Chemistry. 2011; 22:1879-1903. [PubMed: 21830812]

103. Liu Y, et al. Multifunctional $\mathrm{pH}$-sensitive polymeric nanoparticles for theranostics evaluated experimentally in cancer. Nanoscale. 2014; 6:3231-3242. [PubMed: 24500240]

104. Soppimath KS, Aminabhavi TM, Kulkarni AR, Rudzinski WE. Biodegradable polymeric nanoparticles as drug delivery devices. Journal of Controlled Release. 2001; 70:1-20. [PubMed: 11166403]

105. Sengupta $S$, et al. Temporal targeting of tumour cells and neovasculature with a nanoscale delivery system. Nature. 2005; 436:568-572. [PubMed: 16049491]

106. Zhang X, et al. Anti-tumor efficacy and biodistribution of intravenous polymeric micellar paclitaxel. Anti-cancer drugs. 1997; 8:696-701. [PubMed: 9311446]

107. Rupp R, Rosenthal SL, Stanberry LR. VivaGel ${ }^{\text {TM }}$ (SPL7013 Gel): A candidate dendrimer microbicide for the prevention of HIV and HSV infection. International Journal of Nanomedicine. 2007; 2:561-566. [PubMed: 18203424]

108. Eliasof S, et al. Correlating preclinical animal studies and human clinical trials of a multifunctional, polymeric nanoparticle. Proc Natl Acad Sci U S A. 2013; 110:15127-32. [PubMed: 23980155]

109. Pittet L, et al. Development and preclinical evaluation of SEL-068, a novel targeted Synthetic Vaccine Particle (tSVP (TM)) for smoking cessation and relapse prevention that generates high titers of antibodies against nicotine. Journal of Immunology. 2012; 188

110. Davis ME, et al. Evidence of RNAi in humans from systemically administered siRNA via targeted nanoparticles. Nature. 2010; 464:1067-U140. [PubMed: 20305636]

111. Weiss GJ, et al. First-in-human phase 1/2a trial of CRLX101, a cyclodextrin-containing polymercamptothecin nanopharmaceutical in patients with advanced solid tumor malignancies. Invest New Drugs. 2013; 31:986-1000. [PubMed: 23397498]

112. Werner ME, et al. Preclinical Evaluation of Genexol-PM, a Nanoparticle Formulation of Paclitaxel, as a Novel Radiosensitizer for the Treatment of Non-Small Cell Lung Cancer. International Journal of Radiation Oncology Biology Physics. 2013; 86:463-468.

113. Lee KS, et al. Multicenter phase II trial of Genexol-PM, a Cremophor-free, polymeric micelle formulation of paclitaxel, in patients with metastatic breast cancer. Breast Cancer Research and Treatment. 2008; 108:241-250. [PubMed: 17476588]

114. Masserini M. Nanoparticles for Brain Drug Delivery. ISRN Biochemistry. 2013; 2013

115. Foley CP, Nishimura N, Neeves KB, Schaffer CB, Olbricht WL. Real-time imaging of perivascular transport of nanoparticles during convection-enhanced delivery in the rat cortex. Ann Biomed Eng. 2012; 40:292-303. [PubMed: 22009318]

116. Wang YC, et al. Sustained intraspinal delivery of neurotrophic factor encapsulated in biodegradable nanoparticles following contusive spinal cord injury. Biomaterials. 2008; 29:4546-4553. [PubMed: 18774604]

117. Papa S, et al. Selective Nanovector Mediated Treatment of Activated Proinflammatory Microglia/ Macrophages in Spinal Cord Injury. ACS Nano. 2013; 7:9881-9895. [PubMed: 24138479] 
118. Kaur IP, Kakkar S. Nanotherapy for posterior eye diseases. Journal of Controlled Release: Official Journal of the Controlled Release Society. 2014

119. Li H, et al. A PEDF N-terminal peptide protects the retina from ischemic injury when delivered in PLGA nanospheres. Experimental Eye Research. 2006; 83:824-833. [PubMed: 16822505]

120. Alqawlaq S, et al. Preclinical development and ocular biodistribution of gemini-DNA nanoparticles after intravitreal and topical administration: Towards non-invasive glaucoma gene therapy. Nanomedicine: Nanotechnology, Biology and Medicine.

121. Alvarez-Román R, Naik A, Kalia YN, Guy RH, Fessi H. Enhancement of topical delivery from biodegradable nanoparticles. Pharmaceutical research. 2004; 21:1818-1825. [PubMed: 15553228]

122. Jean M, et al. Chitosan-plasmid nanoparticle formulations for IM and SC delivery of recombinant FGF-2 and PDGF-BB or generation of antibodies. Gene Therapy. 2009; 16:1097-1110. [PubMed: 19440230]

123. Kohane DS, et al. Biodegradable polymeric microspheres and nanospheres for drug delivery in the peritoneum. Journal of biomedical materials research. Part A. 2006; 77:351-361. [PubMed: 16425240]

124. Kamei T, et al. Spatial distribution of intraperitoneally administrated paclitaxel nanoparticles solubilized with poly (2-methacryloxyethyl phosphorylcholine-co n-butyl methacrylate) in peritoneal metastatic nodules. Cancer science. 2011; 102:200-205. [PubMed: 20942868]

125. Huang YH, et al. Nanoparticle-delivered suicide gene therapy effectively reduces ovarian tumor burden in mice. Cancer research. 2009; 69:6184-6191. [PubMed: 19643734]

126. Kourtis IC, et al. Peripherally Administered Nanoparticles Target Monocytic Myeloid Cells, Secondary Lymphoid Organs and Tumors in Mice. PLoS ONE. 2013; 8

127. Nagahama R, et al. Nanoparticle-Mediated Delivery of Pioglitazone Enhances Therapeutic Neovascularization in a Murine Model of Hindlimb Ischemia. Arteriosclerosis, Thrombosis, and Vascular Biology. 2012; 32:2427-2434.

128. de Titta A, et al. Nanoparticle conjugation of $\mathrm{CpG}$ enhances adjuvancy for cellular immunity and memory recall at low dose. Proceedings of the National Academy of Sciences of the United States of America. 2013; 110:19902-19907. [PubMed: 24248387]

129. Rothenfluh DA, Bermudez H, O’Neil CP, Hubbell JA. Biofunctional polymer nanoparticles for intra-articular targeting and retention in cartilage. Nature Materials. 2008; 7:248-254.

130. Roa WH, et al. Inhalable nanoparticles, a non-invasive approach to treat lung cancer in a mouse model. Journal of Controlled Release. 2011; 150:49-55. [PubMed: 21059378]

131. Luo Y, et al. An inhalable beta(2)-adrenoceptor ligand-directed guanidinylated chitosan carrier for targeted delivery of siRNA to lung. J Control Release. 2012; 162:28-36. [PubMed: 22698944]

132. Mott B, Thamake S, Vishwanatha J, Jones HP. Intranasal delivery of nanoparticle-based vaccine increases protection against S. pneumoniae. Journal of Nanoparticle Research. 2013; 15:1-7.

133. Malhotra M, Tomaro-Duchesneau C, Saha S, Prakash S. Intranasal delivery of chitosan-siRNA nanoparticle formulation to the brain. Methods in Molecular Biology (Clifton, NJ). 2014; 1141:233-247.

134. Ulery BD, et al. Design of a Protective Single-Dose Intranasal Nanoparticle-Based Vaccine Platform for Respiratory Infectious Diseases. PLoS ONE. 2011; 6

135. Saffran M, et al. A new approach to the oral administration of insulin and other peptide drugs. Science. 1986; 233:1081-4. [PubMed: 3526553]

136. Yun Y, Cho YW, Park K. Nanoparticles for oral delivery: targeted nanoparticles with peptidic ligands for oral protein delivery. Adv Drug Deliv Rev. 2013; 65:822-32. [PubMed: 23123292]

137. des Rieux A, Pourcelle V, Cani PD, Marchand-Brynaert J, Preat V. Targeted nanoparticles with novel non-peptidic ligands for oral delivery. Adv Drug Deliv Rev. 2013; 65:833-44. [PubMed: 23454185]

138. Mane V, Muro S. Biodistribution and endocytosis of ICAM-1-targeting antibodies versus nanocarriers in the gastrointestinal tract in mice. Int J Nanomedicine. 2012; 7:4223-37. [PubMed: 22915850] 
139. Jin Y, et al. Goblet cell-targeting nanoparticles for oral insulin delivery and the influence of mucus on insulin transport. Biomaterials. 2012; 33:1573-82. [PubMed: 22093292] 


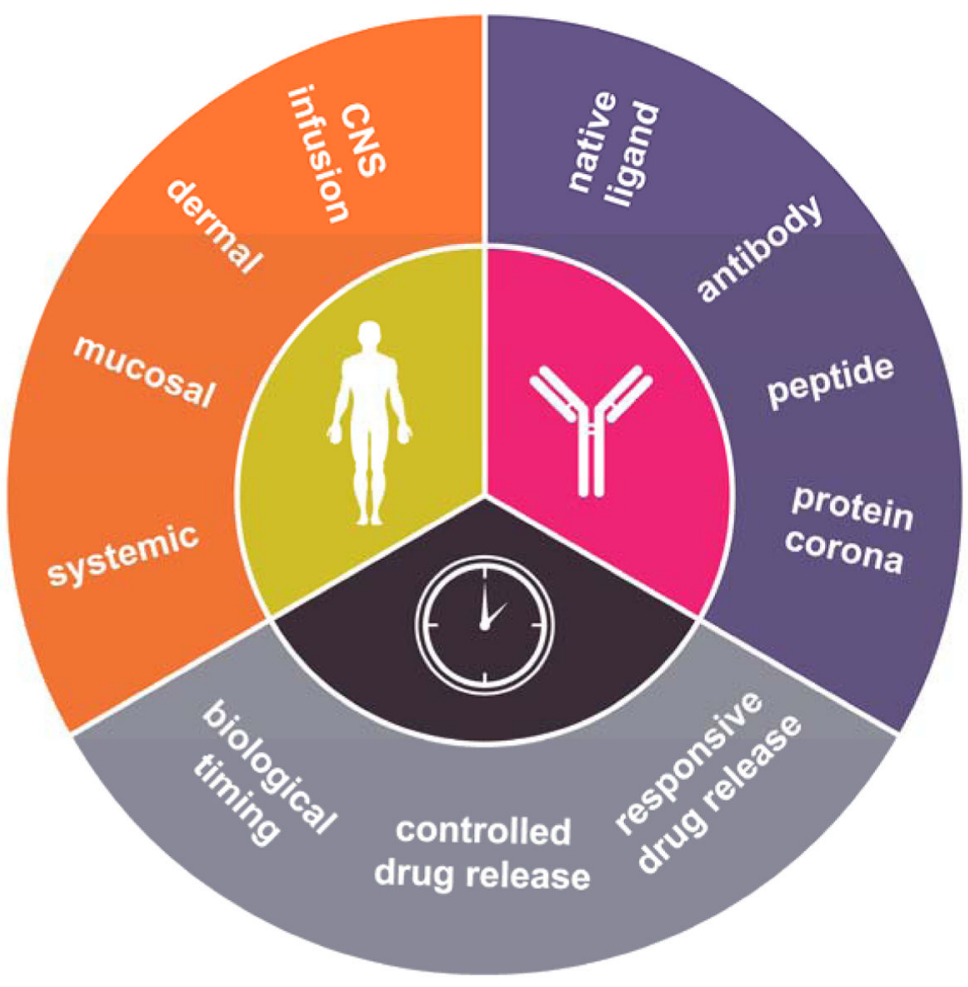

Figure 1.

Holistic perspective of targeting. Representation of key factors influencing nanoparticle targeting organized into anatomical route of delivery, molecular, and temporal aspects. The outer wheel highlights some ways in which nanoparticles can be targeted within the three subgroups. First, different anatomical routes of delivery will affect particle biodistribution providing an initial level of targeting. Second, molecular modifications, either in the form of chemically conjugated targeting ligands and/or proteins adsorbed from the local environment have the capacity to modulate the nature of cellular interactions. Third, the choice of when to treat a disease (biological timing) combined with engineering nanoparticles to have defined drug release profiles (nanoparticle timing) provide an additional mechanism for targeting. Design must be holistic, considering all aspects simultaneously, as the three areas are interconnected; any choice made in one aspect of delivery will have significant effects on the other areas. 
A

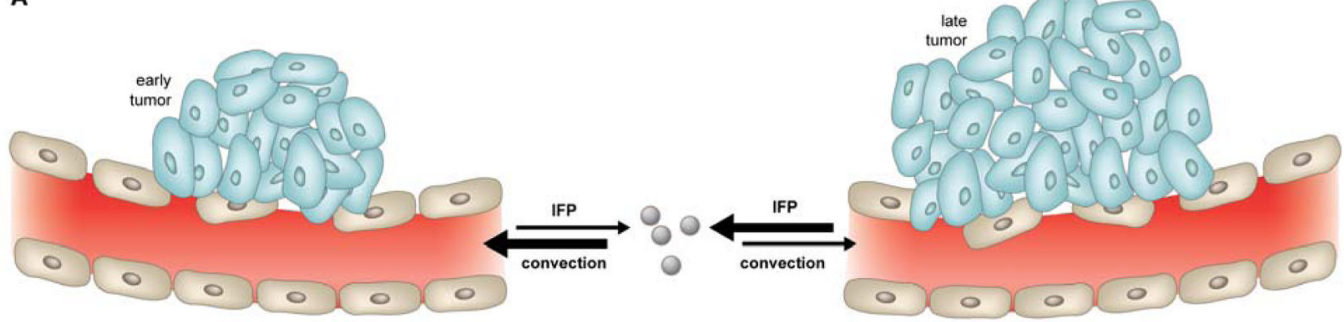

B

mucus
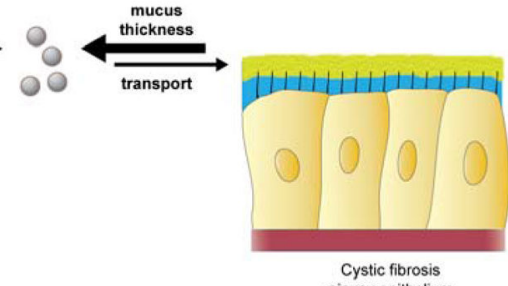

aiway epithelium

Cystic fibrosis
airway epithelium

Figure 2.

Temporal targeting of therapeutic windows. a. As a result of heightened IFP as tumors grow, systemically circulating nanoparticles more readily distribute to early-stage tumors. $\mathbf{b}$.

Mucus thickening is a phenotype of cystic fibrosis that can impede nanoparticle transport. 


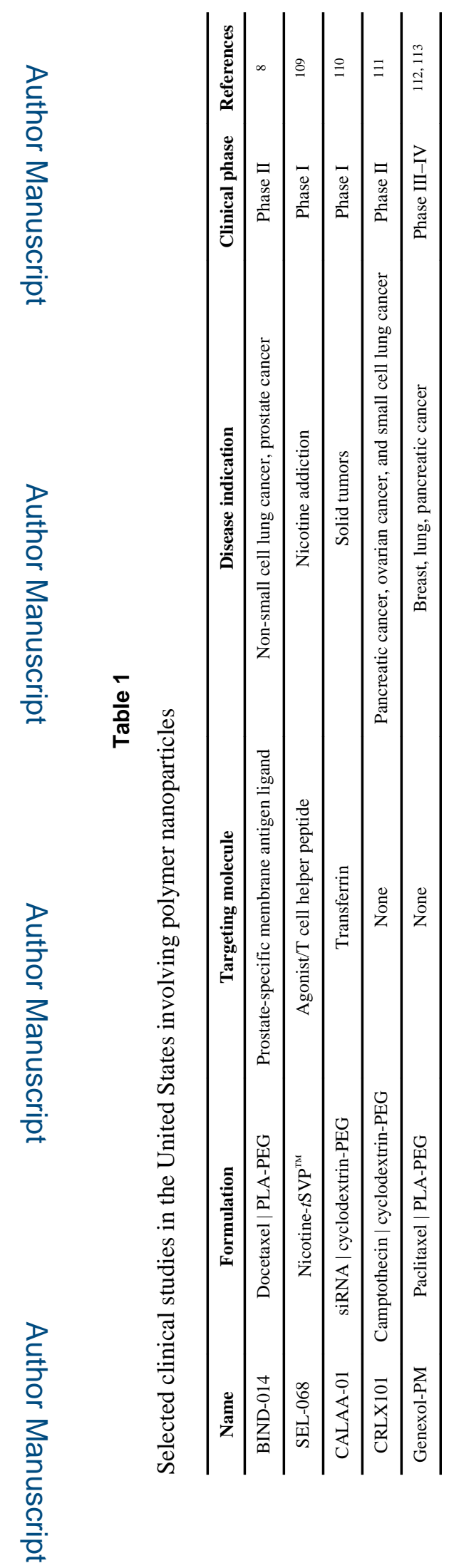

Nat Rev Drug Discov. Author manuscript; available in PMC 2016 April 01. 


\section{Table 2}

Routes of administration for polymer nanoparticles in pre-clinical studies; * indicates incomplete understanding, with more research required.

\begin{tabular}{|c|c|c|c|c|}
\hline Category & Route & $\begin{array}{c}\text { Examples of potential disease } \\
\text { targets }\end{array}$ & Typical Fate & References \\
\hline \multirow{10}{*}{ Non-mucosal local administration } & Brain infusion & $\begin{array}{l}\text { Glioblastoma multiforme, } \\
\text { Pediatric brain tumors, } \\
\text { Parkinson's disease }\end{array}$ & $\begin{array}{c}\text { Size-dependent } \\
\text { penetration, Local } \\
\text { retention, Clearance } \\
\text { by perivascular spaces } \\
\text { possible* }\end{array}$ & $23,24,114,115$ \\
\hline & Spinal cord infusion & Traumatic spinal cord injury & Local retention* & 116,117 \\
\hline & Periocular injection & Glaucoma, retinoblastoma & $\begin{array}{l}\text { Delivery to intraocular } \\
\text { tissues }\end{array}$ & 118 \\
\hline & Intravitreal injection & Macular degeneration & $\begin{array}{l}\text { Delivery to vitreus } \\
\text { chamber and retina }\end{array}$ & $118-120$ \\
\hline & Topical skin application & Insufficient wound healing & Local retention & $18,33,121$ \\
\hline & Subcutaneous injection & $\begin{array}{l}\text { Vaccines for infectious diseases, } \\
\text { lymphoma }\end{array}$ & $\begin{array}{l}\text { Local retention, } \\
\text { Clearance by } \\
\text { lymphatic system }\end{array}$ & $35,36,41,122$ \\
\hline & Intraperitoneal injection & Ovarian cancer & $\begin{array}{l}\text { Retention in } \\
\text { peritoneum, Systemic } \\
\text { distribution }\end{array}$ & $123-125$ \\
\hline & Intramuscular injection & $\begin{array}{c}\text { Ischemia, Vaccines for infectious } \\
\text { diseases }\end{array}$ & $\begin{array}{l}\text { Local retention, } \\
\text { Clearance by } \\
\text { lymphatic system }\end{array}$ & $36,122,126,127$ \\
\hline & Intradermal injection & Vaccines for infectious diseases & $\begin{array}{l}\text { Local retention, } \\
\text { Clearance by } \\
\text { lymphatic system }\end{array}$ & $36,126,128$ \\
\hline & Intra-articular injection & Osteoarthritis & $\begin{array}{l}\text { Size-dependent } \\
\text { penetration, Local } \\
\text { retention, Clearance } \\
\text { by unknown } \\
\text { mechanisms* }\end{array}$ & 129 \\
\hline \multirow{5}{*}{ Mucosal local administration } & Topical vaginal delivery & $\begin{array}{l}\text { Prevention of sexually transmitted } \\
\text { infections }\end{array}$ & $\begin{array}{l}\text { Mucosal retention and } \\
\text { tissue penetration }\end{array}$ & $55,57,58$ \\
\hline & Inhalation delivery & $\begin{array}{l}\text { Cystic fibrosis, Interstitial lung } \\
\text { disease, Lung cancer }\end{array}$ & $\begin{array}{l}\text { Delivery to respiratory } \\
\text { tract }\end{array}$ & 130,131 \\
\hline & Intranasal delivery & Respiratory infectious diseases & $\begin{array}{c}\text { Delivery to respiratory } \\
\text { tract, CNS delivery } \\
\text { possible* }\end{array}$ & $132-134$ \\
\hline & Oral ingestion & Insulin-dependent diabetes & $\begin{array}{l}\text { Delivery to } \\
\text { gastrointestinal tract }\end{array}$ & $135-139$ \\
\hline & Topical eye delivery & Glaucoma & $\begin{array}{l}\text { Delivery to cornea, } \\
\text { Lateral diffusion to } \\
\text { ocular tissues }\end{array}$ & 118,120 \\
\hline Systemic & Intravenous & Diverse & $\begin{array}{l}\text { Systemic distribution, } \\
\text { MPS clearance }\end{array}$ & $4,5,8,95,110$ \\
\hline
\end{tabular}


Table 3

Select challenges facing molecular targeting of polymeric nanoparticles (NP)

\begin{tabular}{|c|c|c|c|}
\hline & Issue & Contributing factors & Potential Solution \\
\hline \multirow{4}{*}{$\begin{array}{c}\text { Factors affecting } \\
\text { targeting specificity }\end{array}$} & Unintended NP uptake & $\begin{array}{c}\text { NP size/shape/charge Cell specific uptake } \\
\text { properties }\end{array}$ & Inert particle coatings (e.g. PEG, HPG) \\
\cline { 2 - 4 } & Protein corona & $\begin{array}{c}\text { Unintentional receptor mediated uptake } \\
\text { Steric hindrance }\end{array}$ & $\begin{array}{c}\text { Composition characterization and } \\
\text { control via NP formulation }\end{array}$ \\
\cline { 2 - 4 } & Receptor identification & Lack of unique targets for cells of interest & Combine with local delivery \\
\hline \multirow{3}{*}{$\begin{array}{c}\text { Factors affecting } \\
\text { therapeutic efficacy }\end{array}$} & Reduced Passive targeting & $\begin{array}{c}\text { Increased immunogenecity Shorter } \\
\text { circulation time }\end{array}$ & $\begin{array}{c}\text { Control ligand density to maximize } \\
\text { avidity and minimize immunogenicity }\end{array}$ \\
\cline { 2 - 4 } & Intracellular fate & $\begin{array}{c}\text { Endosomal entrapment Lysosomal } \\
\text { degredation }\end{array}$ & $\begin{array}{c}\text { Combine with endosomal escape } \\
\text { ligands (e.g. CPP) }\end{array}$ \\
\hline
\end{tabular}

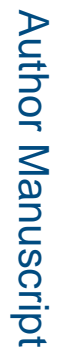

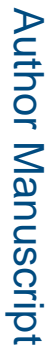

